\title{
VECTOR AND OPERATOR TRAPEZOIDAL TYPE INEQUALITIES FOR CONTINUOUS FUNCTIONS OF SELFADJOINT OPERATORS IN HILBERT SPACES*
}

\author{
SEVER S. DRAGOMIR ${ }^{\dagger}$
}

\begin{abstract}
Some vector and operator generalized trapezoidal inequalities for continuous functions of selfadjoint operators in Hilbert spaces are given. Applications for power and logarithmic functions of operators are provided as well.
\end{abstract}

Key words. Selfadjoint operators, Functions of selfadjoint operators, Spectral representation, Inequalities for selfadjoint operators.

AMS subject classifications. 47A63, 47A99.

1. Introduction. Let $A$ be a selfadjoint linear operator on a complex Hilbert space $(H ;\langle\cdot, \cdot\rangle)$. The Gelfand map establishes an isometric $*$-isomorphism $\Phi$ between the $C^{*}$-algebra $C(S p(A))$ of all continuous functions defined on the spectrum of $A$, denoted by $S p(A)$, and the $C^{*}$-algebra $C^{*}(A)$ generated by $A$ and the identity operator $1_{H}$ on $H$ (see for instance [10, p. 3]) with the property that $\Phi\left(f_{0}\right)=1_{H}$ and $\Phi\left(f_{1}\right)=A$, where $f_{0}(t)=1$ and $f_{1}(t)=t$ for $t \in S p(A)$.

With this notation, we define

$$
f(A):=\Phi(f) \text { for all } f \in C(S p(A))
$$

and we call it the continuous functional calculus for a selfadjoint operator $A$.

If $A$ is a selfadjoint operator and $f$ is a real valued continuous function on $S p(A)$, then $f(t) \geq 0$ for any $t \in S p(A)$ implies that $f(A) \geq 0$, i.e., $f(A)$ is a positive operator on $H$. Moreover, if both $f$ and $g$ are real valued functions on $S p(A)$, then the following important property holds:

$$
f(t) \geq g(t) \text { for any } t \in S p(A) \text { implies that } f(A) \geq g(A)
$$

in the operator order of $B(H)$.

\footnotetext{
${ }^{*}$ Received by the editors on November 29, 2010. Accepted for publication on February 17, 2011. Handling Editor: Moshe Goldberg.

${ }^{\dagger}$ School of Engineering \& Science, Victoria University, PO Box 14481, Melbourne City, MC 8001, Australia (sever.dragomir@vu.edu.au), and School of Computational \& Applied Mathematics, University of the Witwatersrand, Private Bag-3, Wits-2050, Johannesburg, South Africa.
} 
For a recent monograph devoted to various inequalities for continuous functions of selfadjoint operators, see [10] and the references therein.

For other recent results, see [5]-[7], [12]-[14] and [15].

Let $U$ be a selfadjoint operator on the complex Hilbert space $(H,\langle\cdot, \cdot\rangle)$ with the spectrum $S p(U)$ included in the interval $[m, M]$ for some real numbers $m<M$, and let $\left\{E_{\lambda}\right\}_{\lambda}$ be its spectral family. Then for any continuous function $f:[m, M] \rightarrow \mathbb{C}$, it is well known that we have the following spectral representation in terms of the Riemann-Stieltjes integral:

$$
\langle f(U) x, y\rangle=\int_{m-0}^{M} f(\lambda) d\left(\left\langle E_{\lambda} x, y\right\rangle\right)
$$

for any $x, y \in H$. The function $g_{x, y}(\lambda):=\left\langle E_{\lambda} x, y\right\rangle$ is of bounded variation on the interval $[m, M]$, and

$$
g_{x, y}(m-0)=0 \text { and } g_{x, y}(M)=\langle x, y\rangle
$$

for any $x, y \in H$. It is also well known that $g_{x}(\lambda):=\left\langle E_{\lambda} x, x\right\rangle$ is monotonic nondecreasing and right continuous on $[m, M]$.

With the notations introduced above, we have considered in the recent paper [8] the problem of bounding the error

$$
\frac{f(M)+f(m)}{2} \cdot\langle x, y\rangle-\langle f(A) x, y\rangle
$$

in approximating $\langle f(A) x, y\rangle$ by the trapezoidal type formula $\frac{f(M)+f(m)}{2} \cdot\langle x, y\rangle$, where $x, y$ are vectors in the Hilbert space $H$ and $f$ is a continuous function of the selfadjoint operator $A$ with the spectrum in the compact interval of real numbers $[m, M]$.

We recall here only two such results. The first deals with the case of continuous functions of bounded variation and is incorporated in the following theorem [8]:

THEOREM 1.1. Let $A$ be a selfadjoint operator in the Hilbert space $H$ with the spectrum $S p(A) \subseteq[m, M]$ for some real numbers $m<M$, and let $\left\{E_{\lambda}\right\}_{\lambda}$ be its spectral family. If $f:[m, M] \rightarrow \mathbb{C}$ is a continuous function of bounded variation on $[m, M]$, 
then we have the inequalities

$$
\begin{aligned}
& \left|\frac{f(M)+f(m)}{2} \cdot\langle x, y\rangle-\langle f(A) x, y\rangle\right| \\
& \leq \frac{1}{2} \max _{\lambda \in[m, M]}\left[\left\langle E_{\lambda} x, x\right\rangle^{1 / 2}\left\langle E_{\lambda} y, y\right\rangle^{1 / 2}\right. \\
& \left.\quad+\left\langle\left(1_{H}-E_{\lambda}\right) x, x\right\rangle^{1 / 2}\left\langle\left(1_{H}-E_{\lambda}\right) y, y\right\rangle^{1 / 2}\right] \bigvee_{m}^{M}(f) \\
& \leq \frac{1}{2}\|x\|\|y\| \bigvee_{m}^{M}(f)
\end{aligned}
$$

for any $x, y \in H$.

The case of Lipschitzian functions is as follows [8]:

THEOREM 1.2. Let $A$ be a selfadjoint operator in the Hilbert space $H$ with the spectrum $S p(A) \subseteq[m, M]$ for some real numbers $m<M$, and let $\left\{E_{\lambda}\right\}_{\lambda}$ be its spectral family. If $f:[m, M] \rightarrow \mathbb{C}$ is Lipschitzian with the constant $L>0$ on $[m, M]$, then we have the inequalities

$$
\begin{aligned}
\mid & \frac{f(M)+f(m)}{2} \cdot\langle x, y\rangle-\langle f(A) x, y\rangle \mid \\
\leq & \frac{1}{2} L \int_{m-0}^{M}\left[\left\langle E_{\lambda} x, x\right\rangle^{1 / 2}\left\langle E_{\lambda} y, y\right\rangle^{1 / 2}\right. \\
& \left.+\left\langle\left(1_{H}-E_{\lambda}\right) x, x\right\rangle^{1 / 2}\left\langle\left(1_{H}-E_{\lambda}\right) y, y\right\rangle^{1 / 2}\right] d \lambda \\
\leq & \frac{1}{2}(M-m) L\|x\|\|y\|
\end{aligned}
$$

for any $x, y \in H$.

In order to provide error bounds in approximating $\langle f(A) x, y\rangle$ with the quantity

$$
\frac{1}{M-m}[f(m)(M\langle x, y\rangle-\langle A x, y\rangle)+f(M)(\langle A x, y\rangle-m\langle x, y\rangle)]
$$

where $x, y \in H$, which is a generalized trapezoid formula, we obtained in the recent paper [9] the following results:

TheOREM 1.3. Let $A$ be a selfadjoint operator in the Hilbert space $H$ with the spectrum $S p(A) \subseteq[m, M]$ for some real numbers $m<M$, and let $\left\{E_{\lambda}\right\}_{\lambda}$ be its spectral family. 
1. If $f:[m, M] \rightarrow \mathbb{C}$ is continuous and of bounded variation on $[m, M]$, then

$$
\begin{aligned}
& \left|\left\langle\left[\frac{f(m)\left(M 1_{H}-A\right)+f(M)\left(A-m 1_{H}\right)}{M-m}\right] x, y\right\rangle-\langle f(A) x, y\rangle\right| \\
& \leq \sup _{t \in[m, M]}\left[\frac{t-m}{M-m} \bigvee_{m}^{t}\left(\left\langle E_{(\cdot)} x, y\right\rangle\right)+\frac{M-t}{M-m} \bigvee_{t}^{M}\left(\left\langle E_{(\cdot)} x, y\right\rangle\right)\right] \bigvee_{m}^{M}(f) \\
& \leq \bigvee_{m}^{M}\left(\left\langle E_{(\cdot)} x, y\right\rangle\right) \bigvee_{m}^{M}(f) \leq\|x\|\|y\| \bigvee_{m}^{M}(f)
\end{aligned}
$$

for any $x, y \in H$.

2. If $f:[m, M] \rightarrow \mathbb{C}$ is Lipschitzian with the constant $L>0$ on $[m, M]$, then

$$
\begin{aligned}
& \left|\left\langle\left[\frac{f(m)\left(M 1_{H}-A\right)+f(M)\left(A-m 1_{H}\right)}{M-m}\right] x, y\right\rangle-\langle f(A) x, y\rangle\right| \\
& \leq L \int_{m-0}^{M}\left[\frac{t-m}{M-m} \bigvee_{m-0}^{t}\left(\left\langle E_{(\cdot)} x, y\right\rangle\right)+\frac{M-t}{M-m} \bigvee_{t}^{M}\left(\left\langle E_{(\cdot)} x, y\right\rangle\right)\right] d t \\
& \leq L(M-m) \bigvee_{m-0}^{M}\left(\left\langle E_{(\cdot)} x, y\right\rangle\right) \leq L(M-m)\|x\|\|y\|
\end{aligned}
$$

for any $x, y \in H$.

3. If $f:[m, M] \rightarrow \mathbb{R}$ is continuous and monotonic nondecreasing on $[m, M]$, then

$$
\begin{aligned}
& \left|\left\langle\left[\frac{f(m)\left(M 1_{H}-A\right)+f(M)\left(A-m 1_{H}\right)}{M-m}\right] x, y\right\rangle-\langle f(A) x, y\rangle\right| \\
& \leq \int_{m-0}^{M}\left[\frac{t-m}{M-m} \bigvee_{m-0}^{t}\left(\left\langle E_{(\cdot)} x, y\right\rangle\right)+\frac{M-t}{M-m} \bigvee_{t}^{M}\left(\left\langle E_{(\cdot)} x, y\right\rangle\right)\right] d f(t) \\
& \leq \bigvee_{m}^{M}\left(\left\langle E_{(\cdot)} x, y\right\rangle\right)[f(M)-f(m)] \leq\|x\|\|y\|[f(M)-f(m)]
\end{aligned}
$$

for any $x, y \in H$.

For other results of this type, see [9]. For scalar trapezoidal inequalities, see $[1]-[4]$

The main aim of this paper is to provide more results of the above type as well as bounds in the operator order for the selfadjoint linear operator

$$
\left|\frac{f(m)\left(M 1_{H}-A\right)+f(M)\left(A-m 1_{H}\right)}{M-m}-f(A)\right|,
$$

where $f$ is a continuous function of bounded variation, Lipschitzian or convex on $[m, M]$. Applications for certain functions of large interest such as the power and logarithmic functions are provided as well. 
2. Some new vector inequalities. The following result for general continuous functions holds:

ThEOREM 2.1. Let $A$ be a selfadjoint operator in the Hilbert space $H$ with the spectrum $S p(A) \subseteq[m, M]$ for some real numbers $m<M$, and let $\left\{E_{\lambda}\right\}_{\lambda}$ be its spectral family. If $f:[m, M] \rightarrow \mathbb{R}$ is continuous on $[m, M]$, then we have the inequalities

$$
\begin{aligned}
& \left|\left\langle\left[\frac{f(m)\left(M 1_{H}-A\right)+f(M)\left(A-m 1_{H}\right)}{M-m}\right] x, y\right\rangle-\langle f(A) x, y\rangle\right| \\
& \leq\left[\max _{t \in[m, M]} f(t)-\min _{t \in[m, M]} f(t)\right] \bigvee_{m}^{M}\left(\left\langle E_{(\cdot)} x, y\right\rangle\right) \\
& \leq\left[\max _{t \in[m, M]} f(t)-\min _{t \in[m, M]} f(t)\right]\|x\|\|y\|
\end{aligned}
$$

for any $x, y \in H$.

Proof. We observe that, by the spectral representation (1.1), we have the equality

$$
\begin{aligned}
& \left\langle\left[\frac{f(m)\left(M 1_{H}-A\right)+f(M)\left(A-m 1_{H}\right)}{M-m}\right] x, y\right\rangle-\langle f(A) x, y\rangle \\
& =\int_{m-0}^{M} \Phi_{f}(t) d\left(\left\langle E_{t} x, y\right\rangle\right)
\end{aligned}
$$

for any $x, y \in H$, where $\Phi_{f}:[m, M] \rightarrow \mathbb{R}$ is given by

$$
\Phi_{f}(t)=\frac{1}{M-m}[(M-t) f(m)+(t-m) f(M)]-f(t) .
$$

Since $\Phi_{f}$ is continuous on $[m, M]$ and $\Phi_{f}(m)=0$, we can replace in what follows $\int_{m-0}^{M} \Phi_{f}(t) d v(t)$ by $\int_{m}^{M} \Phi_{f}(t) d v(t)$ for every function $v:[m, M] \rightarrow \mathbb{C}$ of bounded variation.

It is well known that if $p:[a, b] \rightarrow \mathbb{C}$ is a continuous function and $v:[a, b] \rightarrow \mathbb{C}$ is of bounded variation, then the Riemann-Stieltjes integral $\int_{a}^{b} p(t) d v(t)$ exists and the following inequality holds:

$$
\left|\int_{a}^{b} p(t) d v(t)\right| \leq \sup _{t \in[a, b]}|p(t)| \bigvee_{a}^{b}(v),
$$

where $\bigvee_{a}^{b}(v)$ denotes the total variation of $v$ on $[a, b]$.

Now, if we denote by $\gamma:=\min _{t \in[m, M]} f(t)$ and by $\Gamma:=\max _{t \in[m, M]} f(t)$, then we have 


$$
\begin{gathered}
\gamma(M-t) \leq(M-t) f(m) \leq \Gamma(M-t) \\
\gamma(t-m) \leq(t-m) f(M) \leq \Gamma(t-m)
\end{gathered}
$$

and

$$
-(M-m) \Gamma \leq-(M-m) f(t) \leq-\gamma(M-m)
$$

for any $t \in[m, M]$. If we add these three inequalities, then we get

$$
-(M-m)(\Gamma-\gamma) \leq(M-m) \Phi_{f}(t) \leq(M-m)(\Gamma-\gamma)
$$

for any $t \in[m, M]$, which shows that

$$
\left|\Phi_{f}(t)\right| \leq \Gamma-\gamma \text { for any } t \in[m, M]
$$

Applying the inequality (2.3) for the representation (2.2), we have from (2.4) that

$$
\left|\int_{m}^{M} \Phi_{f}(t) d\left(\left\langle E_{t} x, y\right\rangle\right)\right| \leq(\Gamma-\gamma) \bigvee_{m}^{M}\left(\left\langle E_{(\cdot)} x, y\right\rangle\right)
$$

for any $x, y \in H$, which proves the first part of (2.1).

If $P$ is a nonnegative operator on $H$, i.e., $\langle P x, x\rangle \geq 0$ for any $x \in H$, then the following inequality is a generalization of the Schwarz inequality in $H$ :

$$
|\langle P x, y\rangle|^{2} \leq\langle P x, x\rangle\langle P y, y\rangle
$$

for any $x, y \in H$.

Now, if $d: m=t_{0}<t_{1}<\cdots<t_{n-1}<t_{n}=M$ is an arbitrary partition of the interval $[m, M]$, then we have by Schwarz's inequality for nonnegative operators that

$$
\begin{aligned}
\bigvee_{m}^{M}\left(\left\langle E_{(\cdot)} x, y\right\rangle\right) & =\sup _{d}\left\{\sum_{i=0}^{n-1}\left|\left\langle\left(E_{t_{i+1}}-E_{t_{i}}\right) x, y\right\rangle\right|\right\} \\
& \leq \sup _{d}\left\{\sum_{i=0}^{n-1}\left[\left\langle\left(E_{t_{i+1}}-E_{t_{i}}\right) x, x\right\rangle^{1 / 2}\left\langle\left(E_{t_{i+1}}-E_{t_{i}}\right) y, y\right\rangle^{1 / 2}\right]\right\}:=I .
\end{aligned}
$$

By the Cauchy-Bunyakovsky-Schwarz inequality for sequences of real numbers, we 
also have that

$$
\begin{aligned}
I & \leq \sup _{d}\left\{\left[\sum_{i=0}^{n-1}\left\langle\left(E_{t_{i+1}}-E_{t_{i}}\right) x, x\right\rangle\right]^{1 / 2}\left[\sum_{i=0}^{n-1}\left\langle\left(E_{t_{i+1}}-E_{t_{i}}\right) y, y\right\rangle\right]^{1 / 2}\right\} \\
& \leq \sup _{d}\left\{\left[\sum_{i=0}^{n-1}\left\langle\left(E_{t_{i+1}}-E_{t_{i}}\right) x, x\right\rangle\right]^{1 / 2}\right\} \sup _{d}\left\{\left[\sum_{i=0}^{n-1}\left\langle\left(E_{t_{i+1}}-E_{t_{i}}\right) y, y\right\rangle\right]^{1 / 2}\right\} \\
& =\left[\bigvee_{m}^{M}\left(\left\langle E_{(\cdot)} x, x\right\rangle\right)\right]^{1 / 2}\left[\bigvee_{m}^{M}\left(\left\langle E_{(\cdot)} y, y\right\rangle\right)\right]^{1 / 2}=\|x\|\|y\|
\end{aligned}
$$

for any $x, y \in H$. These prove the last part of (2.1). $\square$

When the generating function is of bounded variation, we have the following result that complements Theorem 1.3:

ThEOREM 2.2. Let $A$ be a selfadjoint operator in the Hilbert space $H$ with the spectrum $S p(A) \subseteq[m, M]$ for some real numbers $m<M$, and let $\left\{E_{\lambda}\right\}_{\lambda}$ be its spectral family. If $f:[m, M] \rightarrow \mathbb{C}$ is continuous and of bounded variation on $[m, M]$, then we have the inequalities

$$
\begin{aligned}
& \left|\left\langle\left[\frac{f(m)\left(M 1_{H}-A\right)+f(M)\left(A-m 1_{H}\right)}{M-m}\right] x, y\right\rangle-\langle f(A) x, y\rangle\right| \\
& \leq \max _{t \in[m, M]}\left[\frac{M-t}{M-m} \bigvee_{m}^{t}(f)+\frac{t-m}{M-m} \bigvee_{t}^{M}(f)\right] \bigvee_{m}^{M}\left(\left\langle E_{(\cdot)} x, y\right\rangle\right) \\
& \leq \bigvee_{m}^{M}\left(\left\langle E_{(\cdot)} x, y\right\rangle\right) \bigvee_{m}^{M}(f) \leq \bigvee_{m}^{M}(f)\|x\|\|y\|
\end{aligned}
$$

for any $x, y \in H$.

Proof. First of all, observe that

$$
(M-m) \Phi_{f}(t)=(t-M)[f(t)-f(m)]+(t-m)[f(M)-f(t)]
$$

for any $t \in[m, M]$, where here $\Phi_{f}:[m, M] \rightarrow \mathbb{C}$. 
Therefore,

$$
\begin{aligned}
\left|\Phi_{f}(t)\right| & \leq \frac{M-t}{M-m}|f(t)-f(m)|+\frac{t-m}{M-m}|f(M)-f(t)| \\
& \leq \frac{M-t}{M-m} \bigvee_{m}^{t}(f)+\frac{t-m}{M-m} \bigvee_{t}^{M}(f) \\
& \leq \max \left\{\frac{M-t}{M-m}, \frac{t-m}{M-m}\right\}\left[\bigvee_{m}^{t}(f)+\bigvee_{t}^{M}(f)\right] \\
& =\left[\frac{1}{2}+\frac{\left|t-\frac{m+M}{2}\right|}{M-m}\right] \bigvee_{m}^{M}(f)
\end{aligned}
$$

for any $t \in[m, M]$, which implies that

$$
\begin{aligned}
\max _{t \in[m, M]}\left|\Phi_{f}(t)\right| & \leq \max _{t \in[m, M]}\left[\frac{M-t}{M-m} \bigvee_{m}^{t}(f)+\frac{t-m}{M-m} \bigvee_{t}^{M}(f)\right] \\
& \leq \max _{t \in[m, M]}\left[\frac{1}{2}+\frac{\left|t-\frac{m+M}{2}\right|}{M-m}\right] \bigvee_{m}^{M}(f)=\bigvee_{m}^{M}(f) .
\end{aligned}
$$

Applying the inequality (2.3) for the representation (2.2), we have from (2.9) that

$$
\begin{aligned}
\left|\int_{m}^{M} \Phi_{f}(t) d\left(\left\langle E_{t} x, y\right\rangle\right)\right| & \leq \max _{t \in[m, M]}\left[\frac{M-t}{M-m} \bigvee_{m}^{t}(f)+\frac{t-m}{M-m} \bigvee_{t}^{M}(f)\right] \bigvee_{m}^{M}\left(\left\langle E_{(\cdot)} x, y\right\rangle\right) \\
& \leq \bigvee_{m}^{M}(f) \bigvee_{m}^{M}\left(\left\langle E_{(\cdot)} x, y\right\rangle\right)
\end{aligned}
$$

for any $x, y \in H$, which implies the desired result (2.6).

The case of Lipschitzian functions is as follows:

TheOREM 2.3. Let $A$ be a selfadjoint operator in the Hilbert space $H$ with the spectrum $S p(A) \subseteq[m, M]$ for some real numbers $m<M$, and let $\left\{E_{\lambda}\right\}_{\lambda}$ be its spectral family. If $f:[m, M] \rightarrow \mathbb{C}$ is Lipschitzian with the constant $L>0$ on $[m, M]$, then we have the inequalities

$$
\begin{aligned}
& \left|\left\langle\left[\frac{f(m)\left(M 1_{H}-A\right)+f(M)\left(A-m 1_{H}\right)}{M-m}\right] x, y\right\rangle-\langle f(A) x, y\rangle\right| \\
& \leq \bigvee_{m}^{M}\left(\left\langle E_{(\cdot)} x, y\right\rangle\right) \max _{t \in[m, M]}\left[\frac{M-t}{M-m}|f(t)-f(m)|+\frac{t-m}{M-m}|f(M)-f(t)|\right] \\
& \leq \frac{1}{2}(M-m) L \bigvee_{m}^{M}\left(\left\langle E_{(\cdot)} x, y\right\rangle\right) \leq \frac{1}{2}(M-m) L\|x\|\|y\|
\end{aligned}
$$


for any $x, y \in H$.

Proof. We have from the first part of the equality (2.7) that

$$
\begin{aligned}
\left|\Phi_{f}(t)\right| & \leq \frac{M-t}{M-m}|f(t)-f(m)|+\frac{t-m}{M-m}|f(M)-f(t)| \\
& \leq \frac{2 L}{M-m}(M-t)(t-m) \leq \frac{1}{2}(M-m) L
\end{aligned}
$$

for any $t \in[m, M]$, which, by arguments similar to the arguments of the proof of Theorem 2.2, yields the desired result (2.10). The details are omitted.

The following lemma may be stated.

Lemma 2.4. Let $u:[a, b] \rightarrow \mathbb{R}$ and $\varphi, \Phi \in \mathbb{R}$ be such that $\Phi>\varphi$. The following statements are equivalent:

(i) The function $u-\frac{\varphi+\Phi}{2} \cdot e$, where $e(t)=t, t \in[a, b]$, is $\frac{1}{2}(\Phi-\varphi)$-Lipschitzian;

(ii) It holds that

$$
\varphi \leq \frac{u(t)-u(s)}{t-s} \leq \Phi \text { for each } t, s \in[a, b] \text { with } t \neq s
$$

(iii) It holds that

$$
\varphi(t-s) \leq u(t)-u(s) \leq \Phi(t-s) \text { for each } t, s \in[a, b] \text { with } t>s .
$$

Following [11], we can introduce the concept:

Definition 2.5. A function $u:[a, b] \rightarrow \mathbb{R}$ which satisfies one of the equivalent conditions (i)-(iii) is said to be $(\varphi, \Phi)$-Lipschitzian on $[a, b]$.

Notice that in [11], the definition was introduced on utilizing the statement (iii) and only the equivalence (i) $\Leftrightarrow$ (iii) was considered.

Utilizing Lagrange's mean value theorem, we can state the following result that provides practical examples of $(\varphi, \Phi)$-Lipschitzian functions.

Proposition 2.6. Let $u:[a, b] \rightarrow \mathbb{R}$ be continuous on $[a, b]$ and differentiable on $(a, b)$. If

$$
-\infty<\gamma:=\inf _{t \in(a, b)} u^{\prime}(t) \quad \text { and } \sup _{t \in(a, b)} u^{\prime}(t)=: \Gamma<\infty
$$

then $u$ is $(\gamma, \Gamma)$-Lipschitzian on $[a, b]$.

The following corollary holds:

Corollary 2.7. Let $A$ be a selfadjoint operator in the Hilbert space $H$ with the spectrum $S p(A) \subseteq[m, M]$ for some real numbers $m<M$, and let $\left\{E_{\lambda}\right\}_{\lambda}$ be its spectral 
family. If $l, L \in \mathbb{R}$ are such that $L>l$ and $f:[m, M] \rightarrow \mathbb{R}$ is $(l, L)$-Lipschitzian on $[m, M]$, then we have the inequality

$$
\begin{aligned}
& \left|\left\langle\left[\frac{f(m)\left(M 1_{H}-A\right)+f(M)\left(A-m 1_{H}\right)}{M-m}\right] x, y\right\rangle-\langle f(A) x, y\rangle\right| \\
& \leq \frac{1}{4}(M-m)(L-l) \bigvee_{m}^{M}\left(\left\langle E_{(\cdot)} x, y\right\rangle\right) \leq \frac{1}{4}(M-m)(L-l)\|x\|\|y\|
\end{aligned}
$$

for any $x, y \in H$.

Proof. The proof follows by applying the inequality (2.10) to the $\frac{1}{2}(L-l)-$ Lipschitzian function $f-\frac{1}{2}(l+L) e$, where $e(t)=t, t \in[m, M]$. The details are omitted.

When the generating function is continuous convex, we can state the following result as well:

THEOREM 2.8. Let $A$ be a selfadjoint operator in the Hilbert space $H$ with the spectrum $S p(A) \subseteq[m, M]$ for some real numbers $m<M$, and let $\left\{E_{\lambda}\right\}_{\lambda}$ be its spectral family. If $f:[m, M] \rightarrow \mathbb{R}$ is continuous convex on $[m, M]$ with finite lateral derivatives $f_{-}^{\prime}(M)$ and $f_{+}^{\prime}(m)$, then we have the inequalities

$$
\begin{aligned}
& \left|\left\langle\left[\frac{f(m)\left(M 1_{H}-A\right)+f(M)\left(A-m 1_{H}\right)}{M-m}\right] x, y\right\rangle-\langle f(A) x, y\rangle\right| \\
& \leq \frac{1}{4}(M-m)\left[f_{-}^{\prime}(M)-f_{+}^{\prime}(m)\right] \bigvee_{m}^{M}\left(\left\langle E_{(\cdot)} x, y\right\rangle\right) \\
& \leq \frac{1}{4}(M-m)\left[f_{-}^{\prime}(M)-f_{+}^{\prime}(m)\right]\|x\|\|y\|
\end{aligned}
$$

for any $x, y \in H$.

Proof. By the convexity of $f$ on $[m, M]$, we have

$$
f(t)-f(M) \geq f_{-}^{\prime}(M)(t-M)
$$

for any $t \in[m, M]$. If we multiply this inequality by $t-m \geq 0$, then we deduce

$$
(t-m) f(t)-(t-m) f(M) \geq f_{-}^{\prime}(M)(t-M)(t-m)
$$

for any $t \in[m, M]$. Similarly, we get

$$
(M-t) f(t)-(M-t) f(m) \geq f_{+}^{\prime}(m)(M-t)(t-m)
$$

for any $t \in[m, M]$. 
Summing the above inequalities and dividing by $M-m$ we deduce the inequality

$$
\Phi_{f}(t) \leq \frac{(M-t)(t-m)}{M-m}\left[f_{-}^{\prime}(M)-f_{+}^{\prime}(m)\right] \leq \frac{1}{4}(M-m)\left[f_{-}^{\prime}(M)-f_{+}^{\prime}(m)\right]
$$

for any $t \in[m, M]$. By the convexity of $f$, we also have that

$$
\begin{aligned}
\frac{1}{M-m}[(M-t) f(m)+(t-m) f(M)] & \geq f\left(\frac{(M-t) m+(t-m) M}{M-m}\right) \\
& =f(t)
\end{aligned}
$$

giving that

$$
\Phi_{f}(t) \geq 0 \text { for any } t \in[m, M] .
$$

Utilizing (2.3) for the representation (2.2) we deduce from (2) and (2.20) the desired result (2.16).

We observe that a similar result holds for concave functions as well.

3. Inequalities in the operator order. Before we state the next results, we recall that if $A$ is a bounded linear operator, then the operator $A^{*} A$ is selfadjoint and positive and the "absolute value" operator is defined by $|A|:=\sqrt{A^{*} A}$.

In what follows, we use the fact that if $A$ is selfadjoint and $f \in C(S p(A))$, then $|f(A)|=(|f|)(A)$.

The following result providing some inequalities in the operator order may be stated:

THEOREM 3.1. Let $A$ be a selfadjoint operator in the Hilbert space $H$ with the spectrum $S p(A) \subseteq[m, M]$ for some real numbers $m<M$.

1. If $f:[m, M] \rightarrow \mathbb{R}$ is continuous on $[m, M]$, then

$$
\begin{aligned}
& \left|\frac{f(m)\left(M 1_{H}-A\right)+f(M)\left(A-m 1_{H}\right)}{M-m}-f(A)\right| \\
& \leq\left[\max _{t \in[m, M]} f(t)-\min _{t \in[m, M]} f(t)\right] 1_{H} .
\end{aligned}
$$


2. If $f:[m, M] \rightarrow \mathbb{C}$ is continuous and of bounded variation on $[m, M]$, then

$$
\begin{aligned}
& \left|\frac{f(m)\left(M 1_{H}-A\right)+f(M)\left(A-m 1_{H}\right)}{M-m}-f(A)\right| \\
& \leq \frac{M 1_{H}-A}{M-m} \bigvee_{m}^{A}(f)+\frac{A-m 1_{H}}{M-m} \bigvee_{A}^{M}(f) \\
& \leq\left[\frac{1}{2}+\frac{\left|A-\frac{m+M}{2} 1_{H}\right|}{M-m}\right] \bigvee_{m}^{M}(f),
\end{aligned}
$$

where $\bigvee_{m}^{A}(f)$ denotes the operator generated by the scalar function

$$
[m, M] \ni t \longmapsto \bigvee_{m}^{t}(f) \in \mathbb{R}
$$

The same notation applies for $\bigvee_{A}^{M}(f)$.

3. If $f:[m, M] \rightarrow \mathbb{C}$ is Lipschitzian with the constant $L>0$ on $[m, M]$, then

$$
\begin{aligned}
& \left|\frac{f(m)\left(M 1_{H}-A\right)+f(M)\left(A-m 1_{H}\right)}{M-m}-f(A)\right| \\
& \leq \frac{M 1_{H}-A}{M-m}\left|f(A)-f(m) 1_{H}\right|+\frac{A-m 1_{H}}{M-m}\left|f(M) 1_{H}-f(A)\right| \\
& \leq \frac{1}{2}(M-m) L 1_{H} .
\end{aligned}
$$

4. If $f:[m, M] \rightarrow \mathbb{R}$ is continuous convex on $[m, M]$ with finite lateral derivatives $f_{-}^{\prime}(M)$ and $f_{+}^{\prime}(m)$, then we have the inequalities

$$
\begin{aligned}
0 & \leq \frac{f(m)\left(M 1_{H}-A\right)+f(M)\left(A-m 1_{H}\right)}{M-m}-f(A) \\
& \leq \frac{\left(M 1_{H}-A\right)\left(A-m 1_{H}\right)}{M-m}\left[f_{-}^{\prime}(M)-f_{+}^{\prime}(m)\right] \\
& \leq \frac{1}{4}(M-m)\left[f_{-}^{\prime}(M)-f_{+}^{\prime}(m)\right] 1_{H} .
\end{aligned}
$$

Proof. the proof follows by applying the property $(\mathrm{P})$ to the scalar inequalities (2.4), (2.8), (2.11), (2) and (2.20). The details are omitted. $\square$

The following particular case is perhaps more useful for applications: 
Corollary 3.2. Let $A$ be a selfadjoint operator in the Hilbert space $H$ with the spectrum $S p(A) \subseteq[m, M]$ for some real numbers $m<M$. If $l, L \in \mathbb{R}$ with $L>l$ and $f:[m, M] \rightarrow \mathbb{R}$ is $(l, L)$-Lipschitzian on $[m, M]$, then we have

$$
\left|\frac{f(m)\left(M 1_{H}-A\right)+f(M)\left(A-m 1_{H}\right)}{M-m}-f(A)\right| \leq \frac{1}{4}(M-m)(L-l) 1_{H} .
$$

4. More inequalities for differentiable functions. The following result holds:

THEOREM 4.1. Let $A$ be a selfadjoint operator in the Hilbert space $H$ with the spectrum $S p(A) \subseteq[m, M]$ for some real numbers $m<M$. Assume that the function $f:[m, M] \rightarrow \mathbb{C}$ is continuously differentiable on $[m, M]$.

1. If the derivative $f^{\prime}$ is of bounded variation on $[m, M]$, then we have the inequalities

$$
\begin{aligned}
& \left|\left\langle\left[\frac{f(m)\left(M 1_{H}-A\right)+f(M)\left(A-m 1_{H}\right)}{M-m}\right] x, y\right\rangle-\langle f(A) x, y\rangle\right| \\
& \leq \frac{1}{4}(M-m) \bigvee_{m}^{M}\left(f^{\prime}\right) \bigvee_{m}^{M}\left(\left\langle E_{(\cdot)} x, y\right\rangle\right) \\
& \leq \frac{1}{4}(M-m) \bigvee_{m}^{M}\left(f^{\prime}\right)\|x\|\|y\|
\end{aligned}
$$

for any $x, y \in H$.

2. If the derivative $f^{\prime}$ is Lipschitzian with the constant $K>0$ on $[m, M]$, then we have the inequalities

$$
\begin{aligned}
& \left|\left\langle\left[\frac{f(m)\left(M 1_{H}-A\right)+f(M)\left(A-m 1_{H}\right)}{M-m}\right] x, y\right\rangle-\langle f(A) x, y\rangle\right| \\
& \leq \frac{1}{8}(M-m)^{2} K \bigvee_{m}^{M}\left(\left\langle E_{(\cdot)} x, y\right\rangle\right) \\
& \leq \frac{1}{8}(M-m)^{2} K\|x\|\|y\|
\end{aligned}
$$

for any $x, y \in H$.

Proof. First of all we notice that if $f:[m, M] \rightarrow \mathbb{C}$ is continuously differentiable on $[m, M]$, then we have the following representation in terms of the Riemann-Stieltjes integral:

$$
\Phi_{f}(t)=\frac{1}{M-m} \int_{m}^{M} K(t, s) d f^{\prime}(s), \quad t \in[m, M]
$$


where the kernel $K:[m, M]^{2} \rightarrow \mathbb{R}$ is given by

$$
K(t, s):= \begin{cases}(M-t)(s-m) & \text { if } m \leq s \leq t \\ (t-m)(M-s) & \text { if } t<s \leq M .\end{cases}
$$

Indeed, since $f^{\prime}$ is continuously differentiable on $[m, M]$, then the Riemann-Stieltjes integrals $\int_{m}^{t}(s-m) d f^{\prime}(s)$ and $\int_{t}^{M}(M-s) d f^{\prime}(s)$ exist for each $t \in[m, M]$. Now, integrating by parts in the Riemann-Stieltjes integral yields

$$
\begin{aligned}
\int_{m}^{M} K(t, s) d f^{\prime}(s)= & (M-t) \int_{m}^{t}(s-m) d f^{\prime}(s)+(t-m) \int_{t}^{M}(M-s) d f^{\prime}(s) \\
= & (M-t)\left[\left.(s-m) f^{\prime}(s)\right|_{m} ^{t}-\int_{m}^{t} f^{\prime}(s) d s\right] \\
& +(t-m)\left[\left.(M-s) f^{\prime}(s)\right|_{t} ^{M}+\int_{t}^{M} f^{\prime}(s) d s\right] \\
= & (M-t)\left[(t-m) f^{\prime}(t)-(f(t)-f(m))\right] \\
& +(t-m)\left[-(M-t) f^{\prime}(t)+f(M)-f(t)\right] \\
= & (t-m)[f(M)-f(t)]-(M-t)[f(t)-f(m)] \\
= & (M-m) \Phi_{f}(t)
\end{aligned}
$$

for any $t \in[m, M]$, which provides the desired representation (4.3).

Now, utilizing the representation (4.3) and the property (2.3), we have

$$
\begin{aligned}
& \left|\Phi_{f}(t)\right| \\
& =\frac{1}{M-m}\left|(M-t) \int_{m}^{t}(s-m) d f^{\prime}(s)+(t-m) \int_{t}^{M}(M-s) d f^{\prime}(s)\right| \\
& \leq \frac{1}{M-m}\left[(M-t)\left|\int_{m}^{t}(s-m) d f^{\prime}(s)\right|+(t-m)\left|\int_{t}^{M}(M-s) d f^{\prime}(s)\right|\right] \\
& \leq \frac{1}{M-m}\left[(M-t) \bigvee_{m}^{t}\left(f^{\prime}\right) \sup _{s \in[m, t]}(s-m)+(t-m) \bigvee_{t}^{M}\left(f^{\prime}\right) \sup _{s \in[t, M]}(M-s)\right] \\
& =\frac{(t-m)(M-t)}{M-m}\left[\bigvee_{m}^{t}\left(f^{\prime}\right)+\bigvee_{t}^{M}\left(f^{\prime}\right)\right] \\
& =\frac{(t-m)(M-t)}{M-m} \bigvee_{m}^{M}\left(f^{\prime}\right) \leq \frac{1}{4}(M-m) \bigvee_{m}^{M}\left(f^{\prime}\right)
\end{aligned}
$$

for any $t \in[m, M]$.

Using the representation (2.2), we deduce the desired result (4.1). 
Further, we utilize the fact that for an $L$-Lipschitz continuous function, $v$ : $[\alpha, \beta] \rightarrow \mathbb{C}$ and a Riemann integrable function $p:[\alpha, \beta] \rightarrow \mathbb{C}$, the Riemann-Stieltjes integral $\int_{\alpha}^{\beta} p(s) d v(s)$ exists and

$$
\left|\int_{\alpha}^{\beta} p(s) d v(s)\right| \leq L \int_{\alpha}^{\beta}|p(s)| d s .
$$

Then, by utilizing (4.5) we have

$$
\begin{aligned}
& \left|\Phi_{f}(t)\right| \\
& \leq \frac{1}{M-m}\left[(M-t)\left|\int_{m}^{t}(s-m) d f^{\prime}(s)\right|+(t-m)\left|\int_{t}^{M}(M-s) d f^{\prime}(s)\right|\right] \\
& \leq \frac{K}{M-m}\left[(M-t) \int_{m}^{t}(s-m) d s+(t-m) \int_{t}^{M}(M-s) d s\right] \\
& =\frac{K}{M-m}\left[\frac{(M-t)(t-m)^{2}}{2}+\frac{(t-m)(M-t)^{2}}{2}\right] \\
& =\frac{1}{2}(M-m)(t-m)(M-t) K \leq \frac{1}{8}(M-m)^{2} K
\end{aligned}
$$

for any $t \in[m, M]$.

Using the representation (2.2), we deduce the desired result (4.2).

The following inequalities in the operator order are of interest as well:

THEOREM 4.2. Let $A$ be a selfadjoint operator in the Hilbert space $H$ with the spectrum $S p(A) \subseteq[m, M]$ for some real numbers $m<M$. Assume that the function $f: I \rightarrow \mathbb{C}$ with $[m, M] \subset \stackrel{\circ}{I}$ (the interior of $I$ ) is differentiable on $\stackrel{\circ}{I}$.

1. If the derivative $f^{\prime}$ is continuous and of bounded variation on $[m, M]$, then we have the inequalities

$$
\begin{aligned}
& \left|\frac{f(m)\left(M 1_{H}-A\right)+f(M)\left(A-m 1_{H}\right)}{M-m}-f(A)\right| \\
& \leq \frac{\left(A-m 1_{H}\right)\left(M 1_{H}-A\right)}{M-m} \bigvee_{m}^{M}\left(f^{\prime}\right) \\
& \leq \frac{1}{4}(M-m) \bigvee_{m}^{M}\left(f^{\prime}\right) 1_{H} .
\end{aligned}
$$

2. If the derivative $f^{\prime}$ is Lipschitzian with the constant $K>0$ on $[m, M]$, then 
we have the inequalities

$$
\begin{aligned}
& \left|\frac{f(m)\left(M 1_{H}-A\right)+f(M)\left(A-m 1_{H}\right)}{M-m}-f(A)\right| \\
& \leq \frac{1}{2}(M-m)\left(A-m 1_{H}\right)\left(M 1_{H}-A\right) K \\
& \leq \frac{1}{8}(M-m)^{2} K 1_{H} .
\end{aligned}
$$

Proof. The proof follows by the property (P) applied for the scalar inequalities (4.5) and (4.6).

5. Applications for particular functions. It is obvious that the above results can be applied for various particular functions. However, we will restrict here only to the power and logarithmic functions.

1. Consider now the power function $f:(0, \infty) \rightarrow \mathbb{R}, f(t)=t^{p}$ with $p \neq 0$. Applying Theorem 2.8, we can state the following proposition:

Proposition 5.1. Let $A$ be a selfadjoint operator in the Hilbert space $H$ with the spectrum $S p(A) \subseteq[m, M]$ for some real numbers $0<m<M$. Then for any $x, y \in H$, we have the inequality

$$
\begin{aligned}
& \left|\left\langle\left[\frac{m^{p}\left(M 1_{H}-A\right)+M^{p}\left(A-m 1_{H}\right)}{M-m}\right] x, y\right\rangle-\left\langle A^{p} x, y\right\rangle\right| \\
& \leq \frac{1}{4}(M-m) \Delta_{p}\|x\|\|y\|
\end{aligned}
$$

where

$$
\Delta_{p}=p \times \begin{cases}M^{p-1}-m^{p-1} & \text { if } p \in(-\infty, 0) \cup[1, \infty) \\ m^{p-1}-M^{p-1} & \text { if } 0<p<1 .\end{cases}
$$

In particular,

$$
\begin{aligned}
& \left|\left\langle\left[\frac{M\left(M 1_{H}-A\right)+m\left(A-m 1_{H}\right)}{m M(M-m)}\right] x, y\right\rangle-\left\langle A^{-1} x, y\right\rangle\right| \\
& \leq \frac{1}{4} \frac{(M-m)^{2}(M+m)}{m^{2} M^{2}}\|x\|\|y\|
\end{aligned}
$$

for any $x, y \in H$.

The following inequalities in the operator order also hold:

Proposition 5.2. Let $A$ be a selfadjoint operator in the Hilbert space $H$ with the spectrum $S p(A) \subseteq[m, M]$ for some real numbers $0<m<M$. 
If $p \in(-\infty, 0) \cup[1, \infty)$, then

$$
\begin{aligned}
0 & \leq \frac{m^{p}\left(M 1_{H}-A\right)+M^{p}\left(A-m 1_{H}\right)}{M-m}-A^{p} \\
& \leq p \frac{\left(M 1_{H}-A\right)\left(A-m 1_{H}\right)}{M-m}\left(M^{p-1}-m^{p-1}\right) \\
& \leq \frac{1}{4} p(M-m)\left(M^{p-1}-m^{p-1}\right) 1_{H} .
\end{aligned}
$$

If $p \in(0,1)$, then

$$
\begin{aligned}
0 & \leq A^{p}-\frac{m^{p}\left(M 1_{H}-A\right)+M^{p}\left(A-m 1_{H}\right)}{M-m} \\
& \leq p \frac{\left(M 1_{H}-A\right)\left(A-m 1_{H}\right)}{M-m}\left(m^{p-1}-M^{p-1}\right) \\
& \leq \frac{1}{4} p(M-m)\left(m^{p-1}-M^{p-1}\right) 1_{H} .
\end{aligned}
$$

In particular, we have the inequalities

$$
\begin{aligned}
0 & \leq \frac{M\left(M 1_{H}-A\right)+m\left(A-m 1_{H}\right)}{m M(M-m)}-A^{-1} \\
& \leq \frac{\left(M 1_{H}-A\right)\left(A-m 1_{H}\right)}{M-m} \cdot \frac{M^{2}-m^{2}}{m^{2} M^{2}} \\
& \leq \frac{1}{4} \frac{(M-m)^{2}(M+m)}{m^{2} M^{2}} 1_{H} .
\end{aligned}
$$

Proof. The proof follows from (3.4) and the details are omitted.

2. The case of logarithmic function is as follows:

Proposition 5.3. Let $A$ be a selfadjoint operator in the Hilbert space $H$ with the spectrum $S p(A) \subseteq[m, M]$ for some real numbers $0<m<M$. Then for any $x, y \in H$, we have the inequality

$$
\begin{aligned}
& \left|\left\langle\left[\frac{\left(M 1_{H}-A\right) \ln m+\left(A-m 1_{H}\right) \ln M}{M-m}\right] x, y\right\rangle-\langle\ln A x, y\rangle\right| \\
& \leq \frac{1}{4} \frac{(M-m)^{2}}{m M}\|x\|\|y\| .
\end{aligned}
$$

We also have the following inequality in the operator order

$$
\begin{aligned}
0 & \leq \ln A-\frac{\left(M 1_{H}-A\right) \ln m+\left(A-m 1_{H}\right) \ln M}{M-m} \\
& \leq \frac{\left(M 1_{H}-A\right)\left(A-m 1_{H}\right)}{M m} \leq \frac{1}{4} \frac{(M-m)^{2}}{m M} 1_{H} .
\end{aligned}
$$


REMARK 5.4. Similar results can be obtained if ones uses the inequalities from Theorem 4.1 and 4.2. However the details are left to the interested reader.

Acknowledgment. The author would like to thank the anonymous referee for valuable suggestions that have been implemented in the final version of this paper.

\section{REFERENCES}

[1] P. Cerone and S.S. Dragomir. Trapezoidal-type rules from an inequalities point of view. Handbook of Analytic-Computational Methods in Applied Mathematics, G.A. Anastassiou (editor), Chapman \& Hall/CRC Press, New York, 2000, 65-134.

[2] P. Cerone, S.S. Dragomir, and C.E.M. Pearce. A generalised trapezoid inequality for functions of bounded variation. Turkish J. Math., 24(2):147-163, 2000.

[3] S.S. Dragomir. On the trapezoid quadrature formula for Lipschitzian mappings and applications. Tamkang J. Math., 30(2):133-138, 1999.

[4] S.S. Dragomir. An inequality improving the second Hermite-Hadamard inequality for convex functions defined on linear spaces and applications for semi-inner products. J. Inequal. Pure Appl. Math., 3, 2002, Article 3.

[5] S.S. Dragomir. Čebyšev's type inequalities for functions of selfadjoint operators in Hilbert spaces. Preprint, RGMIA Res. Rep. Coll., 11(e), 2008, Article 9. Availiable at http://rgmia.org/issues.php.

[6] S.S. Dragomir. Grüss' type inequalities for functions of selfadjoint operators in Hilbert spaces. Preprint, RGMIA Res. Rep. Coll., 11(e), 2008, Article 11. Availiable at http://rgmia.org/issues.php.

[7] S.S. Dragomir. Inequalities for the Čebyšev functional of two functions of selfadjoint operators in Hilbert spaces. Preprint, RGMIA Res. Rep. Coll., 11(e), 2008, Article 17. Availiable at http://rgmia.org/issues.php.

[8] S.S. Dragomir. Some trapezoidal vector inequalities for continuous functions of selfadjoint operators in Hilbert spaces. Preprint, RGMIA Res. Rep. Coll., 13(2), 2010, Article 14. Availiable at http://rgmia.org/issues.php.

[9] S.S. Dragomir. Some generalized trapezoidal vector inequalities for continuous functions of selfadjoint operators in Hilbert spaces. Preprint, RGMIA Res. Rep. Coll., 13(e), 2010, Article 14. Availiable at http://rgmia.org/issues.php.

[10] T. Furuta, J. Mićić Hot, J. Pečarić, and Y. Seo. Mond-Pečarić Method in Operator Inequalities. Inequalities for Bounded Selfadjoint Operators on a Hilbert Space. Element, Zagreb, 2005.

[11] Z. Liu. Refinement of an inequality of Grüss type for Riemann-Stieltjes integral. Soochow J. Math., 30(4):483-489, 2004.

[12] A. Matković, J. Pečarić, and I. Perić. A variant of Jensen's inequality of Mercer's type for operators with applications. Linear Algebra Appl., 418:551-564, 2006.

[13] B. Mond and J. Pečarić. Convex inequalities in Hilbert spaces. Houston J. Math., 19:405-420, 1993.

[14] B. Mond and J. Pečarić. Classical inequalities for matrix functions. Utilitas Math., 46:155-166, 1994.

[15] J. Pečarić, J. Mićić, and Y. Seo. Inequalities between operator means based on the MondPečarić method. Houston J. Math., 30(1):191-207, 2004. 